\title{
BMJ Open Cardiovascular and cerebrovascular health after pre-eclampsia: the Motherhealth prospective cohort study protocol
}

\author{
Eliza C Miller (1D , ${ }^{1}$ Andrea Miltiades, ${ }^{2}$ Nicole Pimentel-Soler, ${ }^{3}$ Whitney A Booker, ${ }^{3}$ \\ Ruth Landau-Cahana, ${ }^{2}$ Randolph S Marshall, ${ }^{1}$ Mary E D'Alton, ${ }^{3}$ Ronald Wapner, ${ }^{3}$ \\ Kirsten Lawrence Cleary, ${ }^{3,4}$ Natalie Bello ${ }^{5}$
}

To cite: Miller EC,

Miltiades A, Pimentel-Soler N, et al. Cardiovascular and cerebrovascular health after pre-eclampsia: the Motherhealth prospective cohort study protocol. BMJ Open 2021;11:e043052. doi:10.1136/ bmjopen-2020-043052

- Prepublication history and additional material for this paper are available online. To view these files, please visit the journal online (http://dx.doi org/10.1136/bmjopen-2020 043052).

Received 23 July 2020 Revised 04 November 2020 Accepted 14 December 2020

Check for updates

(c) Author(s) (or their employer(s)) 2021. Re-use permitted under CC BY-NC. No commercial re-use. See rights and permissions. Published by BMJ.

For numbered affiliations see end of article.

Correspondence to

Dr Eliza C Miller;

ecm2137@cumc.columbia.edu

\section{ABSTRACT}

Introduction Cardiovascular and cerebrovascular diseases (CCVDs) are the leading cause of maternal mortality in the first year after delivery. Women whose pregnancies were complicated by pre-eclampsia are at particularly high risk for adverse events. In addition, women with a history of pre-eclampsia have higher risk of CCVD later in life. The physiological mechanisms that contribute to increased CCVD risk in these women are not well understood, and the optimal clinical pathways for postpartum CCVD risk reduction are not yet defined. Methods and analysis The Motherhealth Study (MHS) is a prospective cohort study at Columbia University Irving Medical Center (CUIMC), a quaternary care academic medical centre serving a multiethnic population in New York City. MHS began recruitment on 28 September 2018 and will enrol 60 women diagnosed with pre-eclampsia with severe features in the antepartum or postpartum period, and 40 normotensive pregnant women as a comparison cohort. Clinical data, biospecimens and measures of vascular function will be collected from all participants at the time of enrolment. Women in the pre-eclampsia group will complete an additional three postpartum study visits over 12-24 months. Visits will include additional detailed cardiovascular and cerebrovascular phenotyping. As this is an exploratory, observational pilot study, only descriptive statistics are planned. Data will be used to inform power calculations for future planned interventional studies.

Ethics and dissemination The CUIMC Institutional Review Board approved this study prior to initiation of recruitment. All participants signed informed consent prior to enrolment. Results will be disseminated to the clinical and research community, along with the public, on completion of analyses. Data will be shared on reasonable request.

\section{INTRODUCTION}

Cardiovascular and cerebrovascular diseases (CCVDs) are major causes of maternal mortality in the first year after delivery. ${ }^{1}$ Preeclampsia complicates $2 \%-8 \%$ of pregnancies, ${ }^{2}$ and these women are at particularly
Strengths and limitations of this study

- This prospective cohort study collects detailed longitudinal phenotypic data in the postpartum period from women whose pregnancies were complicated by pre-eclampsia with severe features.

- This study represents a multidisciplinary collaboration between clinical researchers in maternal-fetal medicine, neurology, cardiology and anaesthesiology.

- Biospecimens including maternal (blood and cerebrospinal fluid) and fetal (placenta and umbilical cord blood) samples are banked for future analysis.

- This is a single-centre pilot study with limited follow-up, and the comparison cohort of normotensive women is not followed prospectively after the delivery hospitalisation.

high risk of postpartum cardiovascular and cerebrovascular morbidity and mortality. ${ }^{3-8}$ In addition, mounting evidence shows that women with a history of pre-eclampsia have higher risk of CCVD later in life. ${ }^{9-11}$ The physiological mechanisms that contribute to increased CCVD risk in these women are incompletely understood, ${ }^{12-14}$ and optimum clinical pathways for postpartum CCVD risk reduction after pre-eclampsia are not clearly defined. ${ }^{15-17}$ Few prospective studies of women include detailed phenotypic information regarding pregnancy and its complications, and in the majority, the information has been collected retrospectively. ${ }^{18-20}$

An exception is the ongoing prospective Nulliparous Pregnancy Outcomes Study Monitoring Mothers-to-be (nuMoM2b) cohort study, which has already yielded important data regarding the effects of hypertensive pregnancy on future cardiovascular health ${ }^{21}$; however the women in this cohort had limited follow-up and cardiovascular physiological phenotyping in the first several 
years after delivery. ${ }^{22}$ Another cohort of high-risk women at a single centre demonstrated sustained hypertension and abnormal cardiovascular serum biomarkers in the year after delivery. ${ }^{23}$ These studies highlight the fact that significant gaps in knowledge remain regarding the effects of pre-eclampsia on maternal cardiovascular and cerebrovascular physiology in the first year postpartum. Furthermore, the American College of Obstetricians and Gynecologists and the American Heart Association have called for a collaborative approach to help reduce risk of CCVD in women after pregnancy complications. ${ }^{24}$

To address these knowledge gaps, we are conducting the Motherhealth Study (MHS), an observational cohort study of women with and without pre-eclampsia with severe features in the first 12-24 months after delivery. This pilot feasibility study was conceived through ongoing, informal discussions in a multidisciplinary Preeclampsia Working Group at Columbia University Irving Medical Center (CUIMC). This academic forum includes clinicians and researchers of all levels (fellows, junior faculty and senior faculty) from the Departments of Obstetrics and Gynaecology, Anesthesiology, Neurology and Medicine, with the explicit goals of presenting pilot data, brainstorming new hypothesis-driven research and resource sharing. The goal of the MHS is to demonstrate feasibility and collect pilot data to guide effect estimates for the planning of a future larger study involving multiple centres and longerterm follow-up. The study began recruitment on 28 September 2018 and is ongoing, with anticipated completion of recruitment by 31 December 2020 and completion of follow-up visits by 31 December 2022. Here, we describe the MHS methods and protocol.

\section{METHODS AND ANALYSIS}

MHS is a pilot prospective cohort study at CUIMC, an urban quaternary care academic medical centre serving a multiethnic population in New York City. Patients or the public were not involved in the design, or conduct, or reporting or dissemination plans of our research. MHS began recruitment on 28 September 2018 and will enrol a planned 60 women diagnosed at $\geq 20$ weeks gestation or postpartum with pre-eclampsia with severe features, as well as a comparison cohort of a planned 40 pregnant normotensive women. Eligibility criteria are detailed in table 1. Pre-eclampsia is defined as outlined in the 2018 guidelines of the International Society for Study of Hypertension in Pregnancy. ${ }^{25}$ For the purposes of our research protocol, we chose to enrol women who had severe features of pre-eclampsia, with the goal of maximising the likelihood of detecting between-group differences in our outcome measures, and with the recognition that the distinction between 'severe' and 'mild' forms of pre-eclampsia is no longer a preferred approach in clinical practice. Patients with superimposed pre-eclampsia (chronic hypertension with superimposed pre-eclampsia) were eligible for enrolment in the pre-eclampsia group. As this is an exploratory study, the planned sample size of 60 women with pre-eclampsia and 40 normotensive women represents a convenience sample. The primary purpose of our current protocol was to demonstrate feasibility and collect pilot data which will be used to estimate effect sizes for a variety of outcome measures in order to calculate sample sizes for future planned studies. Matching methods were not used. Both cohorts were chosen at random as eligible women were approached for recruitment in order of presentation for clinical care. The study protocol consists of four total visits over 12-24 months for the pre-eclampsia group, and one study visit at the time of the delivery hospitalisation for the normotensive comparison group. As the protocol differs slightly for the two groups, some study procedures are described separately by group.

\section{Eligibility screening}

The screening process for both study groups takes place when a patient is admitted to an outpatient prenatal clinic, inpatient antepartum service, labour and delivery floor or postpartum inpatient unit. An eligibility checklist and screening $\log$ form is completed by study personnel

Table 1 Eligibility criteria for Motherhealth Study

\begin{tabular}{|c|c|c|}
\hline Cohort group & Inclusion criteria & Exclusion criteria* \\
\hline $\begin{array}{l}\text { Pregnant or postpartum women with } \\
\text { PEC with severe features }\end{array}$ & $\begin{array}{l}\text { Pregnant or postpartum women (within } 12 \\
\text { weeks of delivery) } \\
18 \text { years of age or older } \\
\text { PEC (including superimposed PEC) with } \\
\text { severe features as defined by ISSHP } 2018 \\
\text { Guidelines }\end{array}$ & $\begin{array}{l}\text { Contraindication to MRI } \\
\text { Current substance abuse or addiction } \\
\text { Positive SARS-CoV-2 screening on hospital } \\
\text { admission* }\end{array}$ \\
\hline Normotensive pregnant women & $\begin{array}{l}\text { Pregnant women } \\
18 \text { years of age or older } \\
\text { Normotensive }\end{array}$ & $\begin{array}{l}\text { Current substance abuse or addiction } \\
\text { History and/or current diagnosis of PEC } \\
\text { Hypertensive and/or any history of hypertensive } \\
\text { disorder in current and/or previous pregnancy } \\
\text { History of stroke, epilepsy, traumatic brain } \\
\text { injury, multiple sclerosis or other chronic brain or } \\
\text { neurological disease EXCEPT migraine }\end{array}$ \\
\hline
\end{tabular}

${ }^{*}$ Active SARS-CoV-2 infection was added as an exclusion criterion on 3 June 2020 after the COVID-19 pandemic began, due to safety concerns. BP, blood pressure; ISSHP, International Society for Study of Hypertension in Pregnancy; PEC, pre-eclampsia.; 


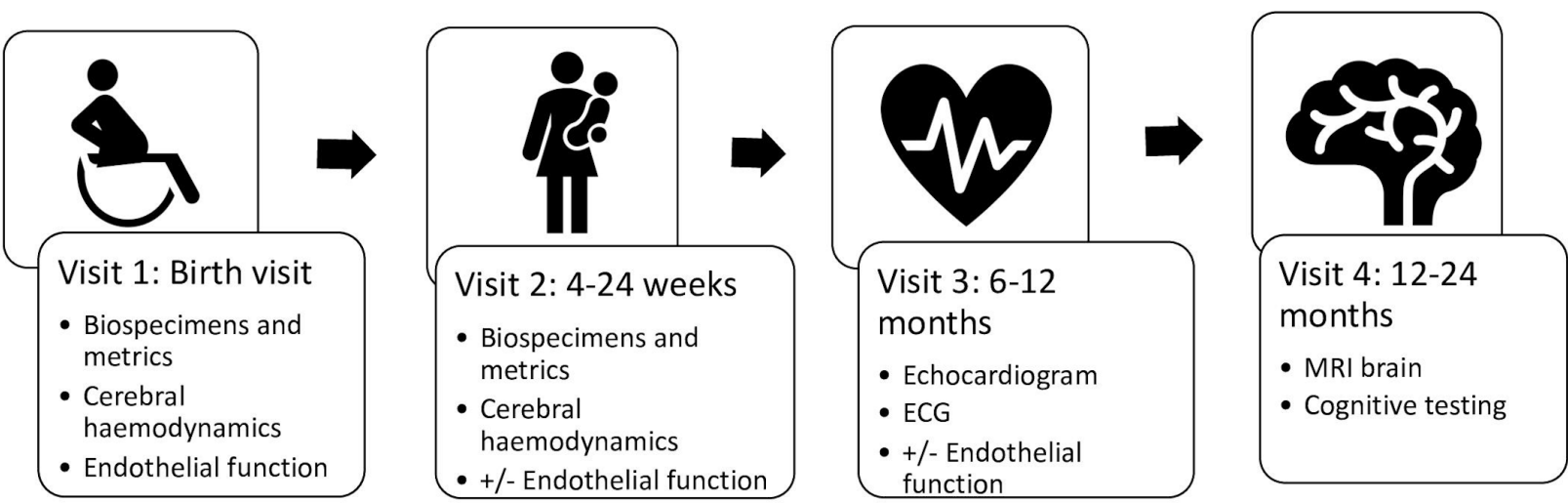

Figure 1 Motherhealth Study visits. Visit 1 ('birth visit') occurs during the delivery hospitalisation, if enrolled antepartum. If enrolled postpartum (ie, participants who first develop pre-eclampsia postpartum and are readmitted), the birth visit may occur up to 12 weeks postpartum and some biospecimens (placenta, cerebrospinal fluid) are not collected. Follow-up endothelial function testing is performed only if initial testing was performed during the birth visit.

while screening the patient's electronic medical record for verification of eligibility criteria. Each screened patient, regardless of cohort group, eligibility or enrolment status generates a study ID number, creating a study record. Eligibility status for consented participants is reviewed and certified by the principal investigator within 1 month of consent. Recruitment tools consisting of study brochures, study information cards and a contact referral form detailing participation procedures and study personnel's contact information are available for both cohort groups.

\section{Patient and public involvement}

Patients or the public were not involved in the design, or conduct, or reporting or dissemination plans of our research.

\section{Overview of study protocol}

Study participation procedures and duration differ by cohort group. The pre-eclampsia group completes a series of up to four visits: a baseline 'birth visit' (V1) and up to three follow-up visits (V2, V3, V4). The normotensive comparison group completes one baseline visit at the time of the delivery hospitalisation (V1). V1 entails similar procedures for both groups, and all participants receive a US $\$ 50.00$ compensation for the completion of V1. Each of the follow-up visits for the pre-eclampsia group addresses a particular aspect of the participant's health (figure 1). The first follow-up visit for the preeclampsia cohort, V2, focuses on postpartum/obstetrical health, and may be merged or take place on the same day as the participant's clinical postpartum visit. It takes place at 4-24 weeks postpartum. The estimated total duration of this follow-up visit is 1.5 hours and participants are compensated US $\$ 50.00$ at the time of the visit. The next follow-up visit for the pre-eclampsia cohort, V3, focuses on cardiovascular health and takes place at 6-12 months postpartum. The estimated total duration of this follow-up visit is 1.5 hours and participants are compensated US $\$ 75.00$ at the time of their visit. The final follow-up visit for the pre-eclampsia cohort, V4, focuses on neurological health and takes place 12-24 months postpartum. The estimated total duration of this follow-up visit is 1.5 hours and participants will be compensated US $\$ 100.00$ at the time of their visit.

Note: A series of up to two consecutive study follow-ups for the pre-eclampsia cohort may be consolidated into a single visit under certain circumstances. This study element was instituted in the setting of the COVID-19 pandemic to limit exposure for study subjects. Elements that are completed at the merged visit and are not conditional on completion at a prior study visit will be completed only once during the merged visit (ie, if a study element is repeated at each of the standalone visits, it is not to be completed twice at the merged visit). Visit duration for merged follow-up visit(s) will depend on the types of visits merged and study visit procedures to be completed. However, it is estimated that no merged follow-up visit will take more than 2.5 hours. Compensation for merged visits will be commensurate with the compensation awarded for each of the standalone visits completed. Estimated duration of total study participation is 12-24 months for the pre-eclampsia group and 96 hours for the normotensive group.

\section{Visit 1 (birth visit) protocol}

The birth visit takes place during a consented participant's hospital admission to the antepartum, labour or postpartum unit. Participants in the pre-eclampsia cohort may be consented any time after 20 weeks gestation and up to 12 weeks postpartum; for participants consented in the postpartum period, timelines are modified and not all birth visit procedures may be completed. Normotensive patients are consented only antepartum or intrapartum.

\section{Birth specimen collection}

Specimen collection for V1 consists of maternal blood, cerebrospinal fluid, placental samples and umbilical cord blood. Each specimen has a specific time frame for collection and processing (table 2). Study personnel will only collect specimens within the denominated time frame. All samples are labelled with study ID number, date and time 
Table 2 Time frame for specimen collection, birth visit

\begin{tabular}{ll} 
Birth specimen type & Time frame for collection \\
\hline Maternal blood & After consent/study enrolment and prior to discharge, preferably intrapartum \\
Placenta & At time of delivery, no later than 2 hours postpartum \\
Cord blood & At time of delivery, no later than 15 min after delivery \\
Cerebrospinal fluid & At time of neuraxial analgesia, if performed (either spinal or combined spinal-epidural) \\
\hline
\end{tabular}

of collection, date and time of processing and specimen type. Data regarding the collection and processing of each specimen are recorded in the study database. Should any sample be removed from the freezer for analysis and/ or thawed for any reason, this is recorded in the study database. Specimen collection protocols are detailed in online supplemental appendix A. Planned analyses include angiogenic profiles, inflammatory and cytokine profiles and biomarkers of cardiovascular disease. In addition, samples will be banked for future use.

\section{Dynamic cerebral autoregulation monitoring}

A 10-minute dynamic cerebral autoregulation study using transcranial Doppler (TCD) and continuous non-invasive blood pressure monitoring using finger plethysmography is completed postpartum for all participants during V1, within 7 days after delivery or at the time of enrolment, if consented after 7 days postpartum. Details of this monitoring protocol have been previously published. ${ }^{26} 27$

\section{Neurologic evaluation}

A brief neurologic evaluation is completed during V1 for all participants, consisting of a focused neurological history, including history of migraines and presence or absence of aura; current neurological symptoms including headache, blurred vision or any other focal symptoms; a baseline modified Rankin scale $(\mathrm{mRS})^{28}$ and a neurological exam, including a standard National Institutes of Health Stroke Scale (NIHSS) ${ }^{29}$ Examinations are completed by a board-certified or board-eligible neurologist certified in administration of the NIHSS and mRS.

\section{Endothelial function assessment}

A non-invasive endothelial function assessment using EndoPat (Itamar Medical) will be performed at the bedside on the postpartum floor 36-72 hours post delivery. Pneumatic probes are fitted on both index fingers. A 5-min brachial artery occlusion is performed with a sphygmomanometer to induce flow-mediated reactive hyperaemia; the ratio of the readings before and after occlusion are then used to calculate the score for endothelial function and reported as the reactive hyperemia index. If a participant is consented during a postpartum admission, they will not complete the assessment. The participant must not be receiving any intravenous medication (eg, magnesium) at the time of the assessment and must fast for 4 hours prior to the assessment. For this reason, these assessments are scheduled early in the morning whenever possible. Additional details of the
EndoPat assessment are included in online supplemental appendix B.

\section{Self-reported measures}

Prior to hospital discharge, all participants complete questionnaires pertaining to the birth visit (online supplemental appendix C). These include self-reported demographic data, medical history and pregnancy history.

\section{Visit 2 protocol (pre-eclampsia group only)}

Participants in the preeclampsia group complete V2 within 4-24 weeks after delivery. This broad time range was adopted to accommodate participants who are enrolled postpartum or who have difficulty with scheduling the visit due to medical issues or family circumstances. The visit includes a series of body measurements including weight, height, waist circumference, hip circumference and blood pressure, following standard procedures and using a validated blood pressure device (Omron). A survey addressing medication intake at the time of visit, breastfeeding status and healthcare utilisation up to time of visit is administered by study personnel and participant responses are recorded in the study database. Survey details are included in online supplemental appendix C. Maternal blood is collected, consisting of two $6 \mathrm{~mL}$ serum separator yellow top tubes. If maternal blood was not collected during V1, a 'catchup collection' consisting of one $6 \mathrm{~mL}$ EDTA purple top tube is collected at the time of this visit in addition to the routine V2. Processing for the $6 \mathrm{~mL}$ EDTA tube will follow steps outlined for the same specimen type in V1. A clean catch urine sample will also be completed at this visit. If endothelial function (EndoPat) testing was completed at V1, a repeat EndoPat test is also performed at V2 using the same protocol. Similarly, if TCD was performed at V1, a repeat TCD is included in V2 using the same protocol.

\section{Visit 3 protocol (pre-eclampsia group only)}

Participants in the pre-eclampsia group complete V3 within 6-12 months after delivery. The visit includes repeat body measurements including weight, height, waist circumference, hip circumference and blood pressure according to the same protocol as V2. If endothelial function (EndoPat) testing was completed at V1 and a repeat test was not completed at V2, a third EndoPat test is performed at V3 using the same protocol. A survey addressing medication intake at the time of visit, breastfeeding status, contraception and healthcare utilisation up to time of visit is administered by study personnel 
and participant responses are recorded in the study database. Survey details are included in online supplemental appendix C. A standard 12 lead ECG and echocardiogram without contrast are obtained according to the American Society of Echocardiography recommendations. ${ }^{30}$

\section{Visit 4 protocol (pre-eclampsia group only)}

Participants in the pre-eclampsia group complete V4 within 12-24 months after delivery. The duration of the visit is expected to be 1.5-2 hours. The visit includes repeat body measurements including weight, height, waist circumference, hip circumference and blood pressure according to the same protocol as V2 and V3. 'Catch-up' procedures including repeat EndoPat testing and maternal blood collection are performed, if needed, according to previously described protocols. A survey addressing medication intake at the time of visit, breastfeeding status, contraception and healthcare utilisation up to time of visit is administered by study personnel and participant responses are recorded in the study database. Survey details are included in online supplemental appendix C. Cognitive testing is performed using the National Institutes of Health (NIH) Toolbox in either English $^{31}$ or Spanish, ${ }^{32}$ depending on the participant's preferred primary language. Tests are administered using a tablet, keyboard and laminated 'home' button sheet according to NIH Toolbox protocol. Cognitive testing includes the following tests from the NIH Toolbox, in the following order: List Sorting Working Memory Test Age 7+v2.1; Pattern Comparison Processing Speech Age 7+v2.1; Flanker Inhibitory Control and Attention Test Age 12+v2.1 and Dimensional Change Card Sort Test Age $12+\mathrm{v} 2.1$. Following cognitive testing, MRI of the brain without contrast enhancement is performed on a 3 Tesla magnet, including the following sequences: diffusionweighted imaging, T1, T2, fluid-attenuated inversion recovery, susceptibility-weighted imaging and magnetic resonance angiography.

\section{DATA ANALYSIS PLAN}

Descriptive statistics will be summarised using percentages for categorical variables and mean (SD) or median (IQR) for continuous variables depending on their distribution. For repeated measures, we will use generalised estimating equations and/or mixed methods to assess changes over time within individual participants. As normotensive women are currently planned to complete one study visit due to funding constraints, only outcome measures from the first study visit will be compared between cohorts. Continuous variables will be compared among women with and without pre-eclampsia using Student's t-tests for normally distributed variables and Wilcoxon rank-sum or Kruskal-Wallis test for non-normally distributed variables. Categorical variables will be compared using $\chi^{2}$ or non-parametric tests as appropriate. Further analysis will include stratification by onset (early onset before 34 weeks gestational age, late onset or postpartum).
ETHICS, SAFETY PROTOCOLS AND DISSEMINATION OF RESULTS

The Institutional Review Board (IRB) approved this study prior to initiation of recruitment. All participants sign informed consent prior to enrolment following all established IRB guidelines. Data are stored on a secure, encrypted online database (Research Electronic Data Capture (REDCap), a secure, web-based application designed to support data capture for research studies) and accessible only to study personnel in accordance with standard protocols. Biospecimens are stored per standard protocols as detailed in online supplemental appendix A and identified by each participant's unique deidentified study ID. Standardised safety protocols have been established for adverse events including elevated blood pressures at follow-up visits, new symptoms reported by the participant and abnormal radiographic findings (online supplemental appendix D). Results will be disseminated to the clinical and research community via abstract presentation at professional meetings and manuscript publication, as soon as analyses are completed. All data, including biospecimens, will be shared on reasonable request from qualified investigators after approval from the study steering committee.

\section{STRENGTHS, LIMITATIONS AND FUTURE DIRECTIONS}

Strengths of the MHS include its multidisciplinary nature and the detailed physiological profiling offering robust pilot data for future studies. Limitations of this pilot study include its small sample size, limiting power to study between-group differences; its limitation to a single academic medical centre, limiting the generalisability of our results; its modest follow-up time of 24 months and its lack of follow-up visits for the control group. The results of this feasibility study will serve to demonstrate effect sizes and help to plan future larger and longer-term studies of the cardiovascular and cerebrovascular effects of pre-eclampsia.

\section{Author affiliations}

${ }^{1}$ Department of Neurology, Columbia University, New York, New York, USA ${ }^{2}$ Department of Anesthesiology, Columbia University, New York, New York, USA ${ }^{3}$ Department of Obstetrics and Gynecology, Columbia University, New York, New York, USA

${ }^{4}$ Department of Obstetrics and Gynecology, Yale University, New Haven, Connecticut, USA

${ }^{5}$ Department of Medicine, Cardiology Division, Columbia University, New York, New York, USA

\section{Twitter Eliza C Miller @ElizaMillerMD}

Acknowledgements The authors acknowledge Michelle DiVito, Caroline Torres and Casandra Almonte for their assistance in the implementation of the study protocol.

Contributors The authors confirm that they have met the following authorship criteria: Substantial contributions to the conception or design of the work (ECM, $\mathrm{AM}, \mathrm{RL}-\mathrm{C}, \mathrm{RSM}, \mathrm{RW}, \mathrm{MED}, \mathrm{KLC}, \mathrm{NB})$, or the acquisition, analysis or interpretation of data (ECM, AM, NP-S, WAB, RSM, NB); drafting the work or revising it critically for important intellectual content (ECM, AM, NP-S, WAB, RL-C, RSM, MED, RW, $\mathrm{KLC}, \mathrm{NB}$ ); gave final approval of the version published (ECM, AM, NP-S, WAB, RL-C, RSM, MED, RW, KLC, NB) and agree to be accountable for all aspects of the work in ensuring that questions related to the accuracy or integrity of any part of the work 
are appropriately investigated and resolved (ECM, AM, NP-S, WAB, RL-C, RSM, MED, $\mathrm{RW}, \mathrm{KLC}, \mathrm{NB})$.

Funding This work is funded by grants from the National Institutes of Health, National Centre for Advancing Translational Sciences (UL1TR001873), the National Institutes of Health, National Institute of Neurological Disorders and Stroke (K23NS107645), the Columbia University Irving Institute Clinical Trials Office, the Louis Katz Foundation (grant number not applicable), and the Louis V. Gerstner Jr Foundation (Gerstner Scholars Programme, grant number not applicable).

Competing interests None declared.

Patient consent for publication Not required

Provenance and peer review Not commissioned; externally peer reviewed.

Supplemental material This content has been supplied by the author(s). It has not been vetted by BMJ Publishing Group Limited (BMJ) and may not have been peer-reviewed. Any opinions or recommendations discussed are solely those of the author(s) and are not endorsed by BMJ. BMJ disclaims all liability and responsibility arising from any reliance placed on the content. Where the content includes any translated material, BMJ does not warrant the accuracy and reliability of the translations (including but not limited to local regulations, clinical guidelines, terminology, drug names and drug dosages), and is not responsible for any error and/or omissions arising from translation and adaptation or otherwise.

Open access This is an open access article distributed in accordance with the Creative Commons Attribution Non Commercial (CC BY-NC 4.0) license, which permits others to distribute, remix, adapt, build upon this work non-commercially, and license their derivative works on different terms, provided the original work is properly cited, appropriate credit is given, any changes made indicated, and the use is non-commercial. See: http://creativecommons.org/licenses/by-nc/4.0/.

\section{ORCID iD}

Eliza C Miller http://orcid.org/0000-0002-7108-8906

\section{REFERENCES}

1 CDC. Pregnancy mortality surveillance system. Centers for Disease Control and Prevention: Reproductive Health, 2016. Available: http:// www.cdc.gov/reproductivehealth/maternalinfanthealth/pmss.html [Accessed 24 Jan 2016].

2 Duley L. The global impact of pre-eclampsia and eclampsia. Semin Perinatol 2009;33:130-7.

3 Hasegawa J, Ikeda T, Sekizawa A, et al. Maternal death due to stroke associated with pregnancy-induced hypertension. Circ $J$ 2015;79:1835-40.

4 Foo L, Bewley S, Rudd A. Maternal death from stroke: a thirty year national retrospective review. Eur J Obstet Gynecol Reprod Biol 2013;171:266-70.

5 Yücesoy G, Ozkan S, Bodur H, et al. Maternal and perinatal outcome in pregnancies complicated with hypertensive disorder of pregnancy: a seven year experience of a tertiary care center. Arch Gynecol Obstet 2005;273:43-9.

6 Sawhney H, Aggarwal N, Biswas R, et al. Maternal mortality associated with eclampsia and severe preeclampsia of pregnancy. $J$ Obstet Gynaecol Res 2000;26:351-6.

7 Alkema L, Chou D, Hogan D, et al. Global, regional, and national levels and trends in maternal mortality between 1990 and 2015 with scenario-based projections to 2030: a systematic analysis by the UN Maternal Mortality Estimation Inter-Agency Group. Lancet 2016;387:462-74.

8 Al-Safi Z, Imudia AN, Filetti LC, et al. Delayed postpartum preeclampsia and eclampsia: demographics, clinical course, and complications. Obstet Gynecol 2011;118:1102-7.

9 Dall'Asta A, D'Antonio F, Saccone G, et al. Cardiovascular events following pregnancies complicated by preeclampsia with emphasis on the comparison between early and late onset forms: a systematic review and meta-analysis. Ultrasound Obstet Gynecol 2020 doi:10.1002/uog.23189. [Epub ahead of print: 02 Jun 2020].

10 Wu P, Haththotuwa R, Kwok CS, et al. Preeclampsia and future cardiovascular health: a systematic review and meta-analysis. Circ Cardiovasc Qual Outcomes 2017;10:e003497.

11 Miller EC, Boehme AK, Chung NT, et al. Aspirin reduces long-term stroke risk in women with prior hypertensive disorders of pregnancy. Neurology 2019;92:e305-16.
12 Cheng S-B, Sharma S. Preeclampsia and health risks later in life: an immunological link. Semin Immunopathol 2016;38:699-708.

13 Hromadnikova I, Kotlabova K, Hympanova L, et al. Cardiovascular and cerebrovascular disease associated microRNAs are dysregulated in placental tissues affected with gestational hypertension, preeclampsia and intrauterine growth restriction. PLoS One 2015;10:e0138383.

14 Sones JL, Davisson RL. Preeclampsia, of mice and women. Physiol Genomics 2016;48:565-72.

15 Bushnell C, McCullough LD, Awad IA, et al. Guidelines for the prevention of stroke in women: a statement for healthcare professionals from the American Heart Association/American Stroke Association. Stroke 2014;45:1545-88.

16 American College of Obstetricians and Gynecologists' Presidential Task Force on Pregnancy and Heart Disease and Committee on Practice Bulletins-Obstetrics. ACOG practice Bulletin No. 212 pregnancy and heart disease. Obstet Gynecol 2019;133:e320-56.

17 Mehta LS, Warnes CA, Bradley E, et al. Cardiovascular considerations in caring for pregnant patients: a scientific statement from the American heart association. Circulation 2020;141:1-20.

18 Tanz LJ, Stuart JJ, Williams PL, et al. Preterm delivery and maternal cardiovascular disease in young and middle-aged adult women. Circulation 2017:135:578-89.

19 Stuart JJ, Tanz LJ, Cook NR, et al. Hypertensive disorders of pregnancy and 10-year cardiovascular risk prediction. J Am Coll Cardiol 2018;72:1252-63.

20 Parikh NI, Jeppson RP, Berger JS, et al. Reproductive risk factors and coronary heart disease in the Women's Health Initiative Observational Study. Circulation 2016;133:2149-58.

21 Haas DM, Parker CB, Marsh DJ, et al. Association of adverse pregnancy outcomes with hypertension 2 to 7 years postpartum. $J$ Am Heart Assoc 2019;8:e013092.

22 Haas DM, Parker CB, Wing DA, et al. A description of the methods of the nulliparous pregnancy outcomes study: monitoring mothers-tobe (nuMoM2b). Am J Obstet Gynecol 2015;212:539.e1-539.e24.

23 Hauspurg A, Countouris ME, Jeyabalan A, et al. Risk of hypertension and abnormal biomarkers in the first year postpartum associated with hypertensive disorders of pregnancy among overweight and obese women. Pregnancy Hypertens 2019;15:1-6.

24 Brown HL, Warner JJ, Gianos E, et al. Promoting risk identification and reduction of cardiovascular disease in women through collaboration with obstetricians and Gynecologists: a presidential Advisory from the American heart association and the American College of obstetricians and Gynecologists. Circulation 2018;137:e843-52.

25 Brown MA, Magee LA, Kenny LC, et al. Hypertensive disorders of pregnancy: ISSHP classification, diagnosis, and management recommendations for international practice. Hypertension 2018;72:24-43.

26 Petersen NH, Ortega-Gutierrez S, Reccius A, et al. Comparison of non-invasive and invasive arterial blood pressure measurement for assessment of dynamic cerebral autoregulation. Neurocrit Care 2014;20:60-8.

27 Miller EC, Dos Santos KRM, Marshall RS, et al. Joint time-frequency analysis of dynamic cerebral autoregulation using generalized harmonic wavelets. Physiol Meas 2020;41:024002-12.

28 van Swieten JC, Koudstaal PJ, Visser MC, et al. Interobserver agreement for the assessment of handicap in stroke patients. Stroke 1988;19:604-7.

29 Meyer BC, Hemmen TM, Jackson CM, et al. Modified National Institutes of health stroke scale for use in stroke clinical trials: prospective reliability and validity. Stroke 2002;33:1261-6.

30 Lang RM, Badano LP, Mor-Avi V, et al. Recommendations for cardiac chamber quantification by echocardiography in adults: an update from the American Society of echocardiography and the European association of cardiovascular imaging. J Am Soc Echocardiogr 2015;28:1-39.

31 Casaletto KB, Umlauf A, Beaumont J, et al. Demographically corrected normative standards for the English version of the NIH Toolbox cognition battery. J Int Neuropsychol Soc 2015;21:378-91.

32 Casaletto KB, Umlauf A, Marquine M, Beaumont J, et al. Demographically corrected normative standards for the Spanish language version of the NIH Toolbox cognition battery. J Int Neuropsychol Soc 2016;22:364-74.

33 Brown MA, Magee LA, Kenny LC, et al. The hypertensive disorders of pregnancy: ISSHP classification, diagnosis \& management recommendations for international practice. Pregnancy Hypertens 2018;13:291-310. 